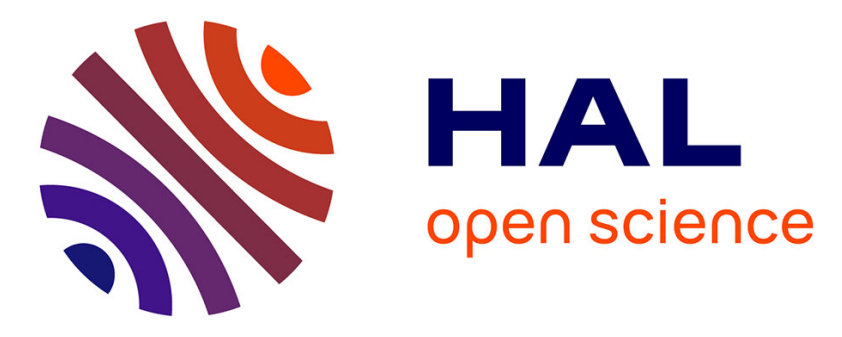

\title{
VASAO: visible all sky adaptive optics: a new adaptive optics concept for CFHT
}

\author{
Olivier Lai, Christian Veillet, Derrick Salmon, Kevin Ho, Marc R. Baril, \\ Gregory A. Barrick, Jim Thomas, Douglas Teeple, Tom Benedict, Jean-Paul \\ Pique, et al.
}

\section{To cite this version:}

Olivier Lai, Christian Veillet, Derrick Salmon, Kevin Ho, Marc R. Baril, et al.. VASAO: visible all sky adaptive optics: a new adaptive optics concept for CFHT. Proceedings of SPIE, the International Society for Optical Engineering, 2008, Adaptive Optics Systems. Edited by Hubin, Norbert; Max, Claire E.; Wizinowich, Peter L., 7015, pp.701543. 10.1117/12.789666 . hal-01118479

\author{
HAL Id: hal-01118479 \\ https://hal.science/hal-01118479
}

Submitted on 4 Mar 2015

HAL is a multi-disciplinary open access archive for the deposit and dissemination of scientific research documents, whether they are published or not. The documents may come from teaching and research institutions in France or abroad, or from public or private research centers.
L'archive ouverte pluridisciplinaire HAL, est destinée au dépôt et à la diffusion de documents scientifiques de niveau recherche, publiés ou non, émanant des établissements d'enseignement et de recherche français ou étrangers, des laboratoires publics ou privés. 


\title{
VASAO: Visible All Sky Adaptive Optics A new adaptive optics concept for CFHT
}

\author{
Olivier Lai ${ }^{\mathrm{a}}$, Christian Veillet ${ }^{\mathrm{a}}$, Derrick Salmon ${ }^{\mathrm{a}}$, Kevin Ho ${ }^{\mathrm{a}}$, Marc R. Baril ${ }^{\mathrm{a}}$, Gregory A. Barrick, \\ Jim Thomas ${ }^{\mathrm{a}}$, Douglas Teeple ${ }^{\mathrm{a}}$, Tom Benedict ${ }^{\mathrm{a}}$, Jean Paul Pique ${ }^{\mathrm{b}}$ Hugues Guillet de Chatellus . $^{\mathrm{b}}$. \\ ${ }^{a}$ Canada France Hawaii Telescope, Kamuela, HI 96743, USA. \\ ${ }^{\mathrm{b}}$ Université Joseph Fourier, Grenoble, France
}

\begin{abstract}
VASAO is an ambitious project that explores new conceptual direction in the field of astronomical adaptive optics. In the era of 8 meter and larger telescopes, and their instrument costs and telescope time pressure, there is a natural niche for such ground-breaking conceptual development in the 4 meter class telescope. The aim of VASAO is to provide diffraction limited imaging in the visible with $100 \%$ sky coverage; the challenge (but potential rewards) arises from the simultaneity of these requirements. To this end, CFHT is conducting a feasibility study based on the polychromatic guide star concept (Foy et al., 1995 [4]) coupled with a high order curvature AO system, presented in this paper.

A number of experiments have been started (or carried out) to study the challenges and limits of the techniques involved in an operational setting; these include the FlyEyes detector, and a polychromatic tip-tilt test on natural stars.

Because such a project straddles such a fine line between facility instrument and experimental facility, careful thought has to be given to the balance between modes of operations and potential astrophysical targets.
\end{abstract}

\section{Introduction}

Current limitations of adaptive optics are constantly being pushed back with innovative ideas and technology. For example, the limited corrected (isoplanatic) field of view is being expanded by techniques such as MCAO, MOAO and GLAO for various astrophysical applications. Dynamic range and extension to shorter wavelengths are being studied for extreme $\mathrm{AO}$ and high order $\mathrm{AO}$; visible adaptive optics on large telescopes is conceptually not that challenging, but will be challenging from a technical standpoint. Yet the returns are large, as the resolution decreases in direct proportionality with the wavelength. Sky coverage has been dramatically expanded with the advent of laser guide stars. Yet, one vexing problem still remains with regards to sky coverage, and that is the tip-tilt determination problem. The equivalent return path of light means that laser guide stars cannot provide information about the overall tip-tilt, and many schemes have been studied to circumvent this problem. The most promising of these is the polychromatic laser guide star, proposed by Renaud Foy et al. (1995 [4]), and currently being demonstrated in the ELP-OA project.

Sky coverage is indeed an important issue for adaptive optics as specific objects of interest may simply be too faint, or in certain cases such as the various deep fields, regions of the sky are selected for their lack of bright star in the vicinity. Therefore, the VASAO concept was developed (Veillet et al., 2006 [14]) to study the possibility of providing visible AO correction over the entire sky.

\section{Test bench for advanced concepts}

The 2-4meter class telescopes are in a precarious position in the era of 8-10meter telescopes and the planning stages of 30-40meter telescopes. However, there is a way to capitalize on the investment made on these observatories. This requires some risk-taking and using these telescopes at the forefront of the technology. Thus in a sense they become test-benches for advanced concepts, but in and of itself this is not sufficient to justify the risks; there also needs to be a astrophysical niche to motivate a dual approach (both technical and scientific), that eventually enables some capability unavailable elsewhere. In this light, the VASAO concept would provide a powerful test bench for laser guide stars and 40 milli-arcsecond resolution anywhere in the sky in the visible.

This paper is arranged as follows: Section 2 describes some sample astrophysical applications that would be enabled by such an instrument. Section 3 describes the various components and sub-systems required and section 4 is a presentation of the system wide feasibility study. Conclusion and future ideas for research are presented in section 5 .

Adaptive Optics Systems, edited by Norbert Hubin, Claire E. Max, Peter L. Wizinowich, Proc. of SPIE Vol. 7015, 701543, (2008)

0277-786X/08/\$18 $\cdot$ doi: $10.1117 / 12.789666$

Proc. of SPIE Vol. 7015 701543-1 


\section{Science cases}

VASAO is proposed as a general-purpose instrument, and many astrophysical topics would benefit from diffraction limited imaging in the visible without the need for a reference source. The main limitation is of course the corrected field of view that becomes smaller with wavelength. VASAO would be a single object imager/spectrograph, but in order to obtain proper sampling (15mas pixels), the spatial dynamic range would in fact remain unchanged with conventional near-infrared correction (see Figure 2).

\subsection{Point source sensitivity}

From a simple extrapolation of MegaCam's point source sensitivity, we find that the VASAO sensitivity would indeed be excellent. As shown in Table 1, we start from MegaCam zero points, and we correct for the throughput, measured on MegaCam, estimated on VASAO. We note that VASAO would use a red-optimized detector, which allows regaining some of the sensitivity at longer wavelengths. We then apply a correction for the fact that only a fraction of the flux (equal to the Strehl ratio) will be in the coherent core and this determines the aperture used in VASAO. Eventually we find that in R band, a magnitude of 27.5 can be detected at the $5 \sigma$ level in one hour of exposure in dark sky conditions. This opens the way to many astrophysical applications.

Table 1: Estimating the VASAO point source sensitivity from MegaCam's zero point and throughput.

\begin{tabular}{|c|c|c|c|c|}
\hline Wavelength $(\lambda, \mu \mathrm{m})$ & 0.487 & 0.625 & 0.770 & 0.900 \\
\hline MegaCam zero point & 26.96 & 26.47 & 26.24 & 25.30 \\
\hline MegaCam throughput & 0.568 & 0.470 & 0.366 & 0.173 \\
\hline VASAO throughput & 0.262 & 0.298 & 0.273 & 0.176 \\
\hline zero point correction & -0.803 & -0.457 & -0.281 & 0.05 \\
\hline VASAO aperture radius & 31 mas & 36 mas & 43 mas & 58 mas \\
\hline VASAO Strehl & 0.26 & 0.38 & 0.5 & 0.68 \\
\hline zero point add for SNR(gain) & 1.31 & 1.56 & 1.67 & 1.68 \\
\hline $\begin{array}{c}\text { VASAO zero point gain } \\
\text { (1hoint source detection limit }\end{array}$ & 0.51 & 1.11 & 1.39 & 1.73 \\
\hline
\end{tabular}

\subsection{Sample astrophysical targets}

A few examples of astrophysical problems that could benefit from the VASAO system are given. While this list is by no means exhaustive, it illustrates the potential of the instrument and shows that studying individual objects can provide answers to deep astrophysical problems.

\subsubsection{Solar system}

The study of binarity of asteroids, trans-neptunians and minor bodies allows to probe the composition, thus the history and evolution of the solar system. Traditionally, observations of such objects is limited to bright objects or appulses, which sets severe time constraints. With the help of VASAO, any object of any brightness can be observed at any moment in time, opening the way to surveys of these objects without any bias on size or distance.

\subsubsection{YSOs}

Young stellar objects are often in regions of high absorption (e.g. Taurus, Orion); therefore, sufficiently bright guide stars are not always available. Furthermore, the structures (disks, etc.) around YSOs are usually at a high contrast so even in the infrared, the stable, high Strehl PSF delivered by VASAO would be beneficial to extend existing work (e.g. Ménard et al., 1999 [7]). Also, in the visible, in particular in narrow lines of oxygen, astrophysical jets can be imaged in great detail; their time evolution can provide insights into the turbulence involved in shocks (e.g. Dougados et al., 2000 [3]). 


\subsubsection{Quasar host galaxies}

The study of star formation in quasar host galaxies benefits from VASAO in two ways. First and foremost, detecting the quasar host galaxy is often difficult due to the high contrast required to detect such faint and extended structures around bright point-like sources. Adaptive optics can be very useful in this case as it allows spacially separating the contribution of the quasar from its host galaxy, whether in the near infrared where the high strehl, stable PSF can easily be subtracted or in the visible, where the enhanced resolution concentrates the flux from the unresolved quasar in a few pixels. Second, the star-forming regions within the host galaxy are most likely unresolved, hence they benefit from the increased sensitivity point source sensitivity with respect to the resolved background host galaxy emission (Steinbring, private communication).

\subsubsection{Deep Fields}

Deep fields (e.g. GEMS, HUDF) were chosen specifically for their lack for bright stars. This means that the only way to obtain high angular resolution images of deep fields from the ground will require solving the tip-tilt determination problem. VASAO would thus allow following up individual objects, such as primordial galaxies, with exquisite resolution. The visible domain is especially enticing as it would allow probing the red-shifted UV and would offer glimpses of star formation in early, interacting galaxies.

At this point we introduce the idea that being able to obtain diffraction limited imaging in the near-IR anywhere in the sky is also very valuable from a scientific point of view and drastically relaxes the tolerances on the tip-tilt measurement. Another possible reduced goal or intermediate phase would be to provide diffraction limit in the visible but in the vicinity of a sufficiently bright star to provide the tip-tilt $(<12)$, as we will see in the next sections that the polychromatic LGS tip-tilt measurement is the largest term in the error budget and the biggest challenge to obtaining the diffraction limit at short wavelengths.

\section{Instrumental Concept}

The VASAO concept can be broken down into two almost independent sub-systems: The high order AO providing the diffraction limit of the telescope in the visible and the tip-tilt/guiding which stabilizes the image and allows guiding in open loop with respect to the sky. The high order AO system requires a laser guide star, but this can be conventional (alternately some of the light from the polychromatic LGS can be used for the high order wavefront sensing). The tiptilt/guider requires a hierarchy of devices to correct for the atmosphere at a frequency of $\sim 10 \mathrm{~Hz}$, the mechanical guiding $(\sim 0.1 \mathrm{~Hz})$ and the drift of the telescope (order of minutes). Respectively, these are the polychromatic laser guide star, which obtains the tip-tilt information from the chromatic differential tip-tilt, a seismometer to maintain the telescope's attitude in an inertial frame of reference and a slow guider which is used to counteract drifts and can be obtained from a much wider field (or non-destructive reads of the science detector).

\subsection{High order AO system PUEO HOU}

With the idea of upgrading PUEO, the CFHT 19 element curvature adaptive optics Bonnette, and due to the experience available at the University of Hawaii, an 85 sub-apertures/electrodes curvature system (PUEO HOU) was suggested as a starting point. Also, previous simulations of relatively high order curvature systems for 4 meter class telescopes (Lai, 2002 [6]) suggested that it was not necessary to increase the number of degrees of freedom beyond 85 to provide diffraction limited performance in the visible domain. From a technical standpoint such a system is not a major challenge and 85-element curvature adaptive optics have successfully been validated on the sky (e.g. Hokupa'a-85, NICI)

\subsubsection{FlyEyes}

An alternative to APDs as a detector for curvature wavefront sensing was developed at ESO and has been tested at CFHT (Ho et al., 2006, and references therein [5]). It is based on the CCID-35 detector (Beletic et al, 2000 [2]). Ongoing sky tests have revealed that the performance in the bright guide star regime is unaltered and the temporal error introduced by the read-out of the detector is negligible. Figure 1shows characterization data from early engineering in 1996, and the data points obtained in April 2007 with the FlyEyes detector.

Data was obtained on a range of magnitudes with both the APDs and FlyEyes, but varying seeing conditions and especially turbulence correlation times has so far prevented a quantitative analysis of performance as a function of 
guide star brightness. Anecdotally, a 15.8 magnitude star observed with FlyEyes at $250 \mathrm{~Hz}$ provided a $25 \%$ Strehl ratio in $\mathrm{K}^{\prime}$ band. We note in passing that the high order correction requires fewer photons than the measurement of the differential tip-tilt, as it dominates the error budget (see section 4.1). Therefore the data presented on Figure 1, which shows that performance in the bright guide star regime is not degraded with respect to APDs, demonstrates that FlyEyes is perfectly adequate for use with VASAO.
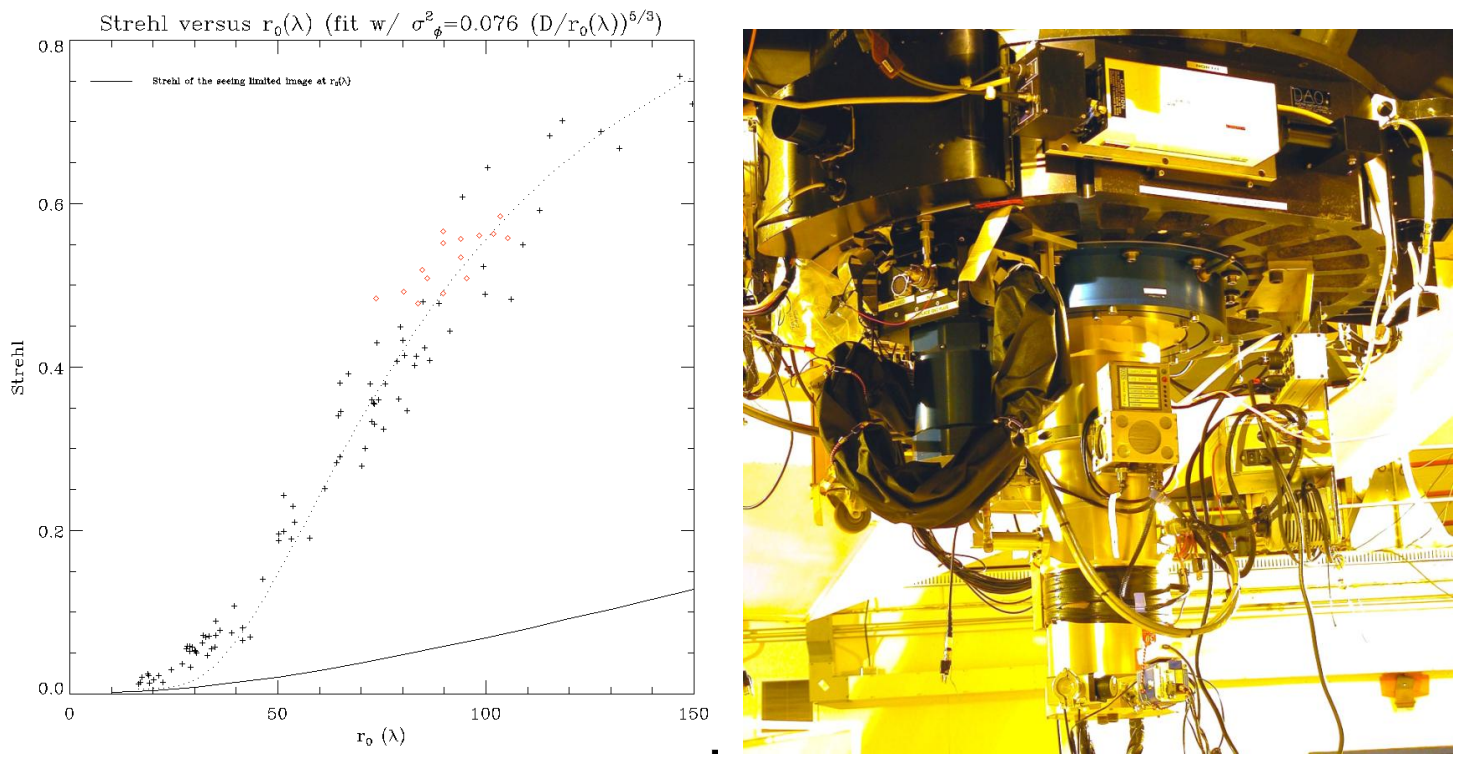

Figure 1. Left: PUEO characterization data (crosses) with April 2007 FlyEyes data (red diamonds). Right: FlyEyes dewar (left) mounted on AOB (top), mounted next to KIR infrared array (gold dewar in center).
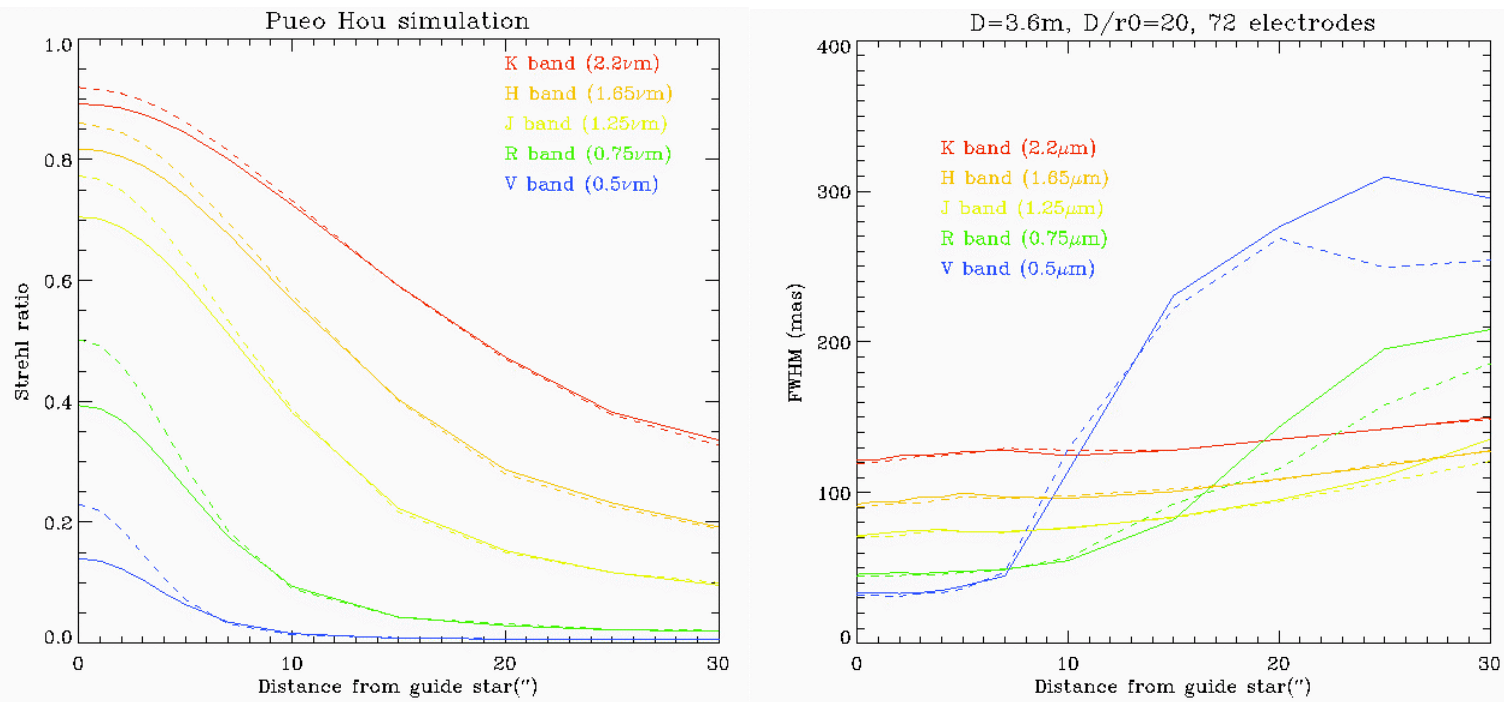

Figure 2: Strehl ratio (left) and FWHM (right) as a function of distance to the guide star. The dotted line is for a natural guide stars and the full line for an LGS.

\subsubsection{Performance from Monte Carlo simulations}

Simulations were carried out using yao, a yorick program developed and maintained by François Rigaut (private communication [9]). The Monte Carlo simulations of an 85 sub-apertures/electrodes curvature system were carried out for a $\mathrm{D} / \mathrm{r}_{0}$ of 20 with a natural and laser guide star and was found to provide adequate performance for the visible 
domain. Results are shown on Figure 2. The high strehl ratio in the near-IR will provide a stable PSF, while in the visible, the resolution will be better than 50 mas over 20 " diameter fields.

\subsubsection{Focal anisoplanatism}

On Figure 2, it can be seen that focal anisoplanatism is not limiting factor for a 4 meter class telescope, even in the visible. Also, because of the smaller $\mathrm{D} / \mathrm{r}_{0}$, the constraint on the radius of curvature is relaxed and the success of Hokupa'a and NICI show that such a system would pose no particular technical challenge. The laser guide star could either be a dedicated conventional solid state laser tuned to the $\mathrm{D}_{2}$ line at $589 \mathrm{~nm}$, or some light of the polychromatic laser could be diverted for the wavefront sensing. Due to the efficiency of curvature sensing and the large sub-apertures the power requirements are comparable to those of existing laser guide stars on $8 \mathrm{~m}$ telescopes.

\subsection{Polychromatic Laser Guide Star}

The tip-tilt determination problem stems from the fact that the laser beam propagating upwards to create the artificial source required for wavefront sensing suffers the same overall tip-tilt on its downward travel, and so, as seen from the ground, it appears fixed on the sky: the tip-tilt information is lost. The principle of the polychromatic laser guide star was introduced by Foy et al. (1995 [4]) and uses the fact that the refractive index of air is chromatic, so that any linear function of the wavefront can be extracted from its differential (chromatic) effect.

\subsubsection{Principle}

Measuring the absolute tip-tilt from its differential chromatic effect is difficult because it requires measuring a second order effect, which is usually neglected in adaptive optics. If we can write that the refractive index $n(\lambda, P, T)=f(\lambda) g(P, T)$ then:

$$
\frac{n\left(\lambda_{1}, P, T\right)-n\left(\lambda_{2}, P, T\right)}{n\left(\lambda_{3}, P, T\right)}=\frac{\Delta n}{n-1}=\frac{F\left(\lambda_{1}\right)-F\left(\lambda_{2}\right)}{F\left(\lambda_{3}\right)}
$$

where $F(\lambda)$ can be any linear function of the wavefront (e.g. Zernike mode such as tip or tilt). Therefore:

$$
\theta=\Delta \theta \frac{n-1}{\Delta n}
$$

This equation states that the absolute tip-tilt is equal to the differential tip-tilt multiplied by the factor $(n-1) / \Delta n$. We would like this factor to be as small as possible to relax the accuracy required on the differential tip-tilt measurements, therefore $\Delta n$ to be as large as possible. As the chromatic dependence of the refractive index of air mostly occurs at short wavelengths, one of the chromatic images will be obtained in the UV, and for the greatest leverage, one would ideally measure the other chromatic image in the infrared, although the chromatic variation of the refractive index is almost flat beyond $600 \mathrm{~nm}$. Thus, in practice, the sodium UV-D2 line at $330 \mathrm{~nm}$ and the doublet at $589 \mathrm{~nm}$ are ideal candidates, yielding a $(n-1) / \Delta n$ on the order of 25 . This means that the accuracy required on the each of the chromatic images has to be $\sqrt{2} \times 25$ smaller than the required accuracy on the absolute tip-tilt. For example, if we require the absolute tip-tilt to be less than the FWHM in the visible, which on a 4 meter telescope is $\sim 30$ mas, the centroid of each spot has to be measured with a precision better than 1 mas. In fact, if we assume that the FWHM of each spot, $\sigma_{s p o t}$ is the same (e.g. limited by seeing), we can write:

$$
\sigma_{\theta}=\frac{n-1}{\Delta n}\left[\frac{\sigma_{\lambda_{1}}}{\sqrt{N_{\lambda_{1}}}}+\frac{\sigma_{\lambda_{2}}}{\sqrt{N_{\lambda_{2}}}}\right]=25 \sqrt{2} \frac{\sigma_{\text {spot }}}{\sqrt{N}}
$$

\subsubsection{Spot size and brightness}

\subsubsection{Required spot size as a function of return photons}

Assuming it is possible to obtain 2 photons $/ \mathrm{cm}^{2} / \mathrm{s} / \mathrm{W}$ return from the sodium layer, and integration times of $50 \mathrm{msec}$ do not introduce a severe servo-lag error, obtaining a residual error of 1 mas requires an equivalent magnitude 11 (roughly $20 \mathrm{~W}$ of laser power) if the spot has a FWHM of 0.14", but an equivalent magnitude of 7 (approximately 930W) if the spot is seeing and optics limited with a FWHM of 1". In practice, it is likely that the 50msec integration time will incur a non-negligible servo-lag error, and reducing the integration time linearly increases the number of photons (thus the 
laser power required. This is illustrated in Figure 3, which shows the Strehl attenuation due to tip-tilt only as a function of wavelength for various spot sizes $\left(0.12 ", 0.4 ", 1.0\right.$ " and 1.4 ") and turbulence strengths $\left(\mathrm{r}_{0}=0.25 \mathrm{~m}, 0.20 \mathrm{~m}, 0.16 \mathrm{~m}\right.$, $0.12 \mathrm{~m}$ ). These curves assume $4.10^{5}$ return photons $/ \mathrm{s} / \mathrm{m}^{2}$ (Schöck et al., 2002 [11]), which would be equivalent to a $20 \mathrm{~W}$ laser in the case of 2 photons $/ \mathrm{cm}^{2} / \mathrm{s} / \mathrm{W}$. Although UV-D2 excitation at 330nm shows much promise in improving return fluxes, it illustrates the importance of obtaining a small spot size for a given number of return photons; this is not an easy task. Simulations show that an up-launch AO correction would provide some limited correction; it is quite photon starved, the correction has to be done at very short wavelength and it has never been successfully achieved before.

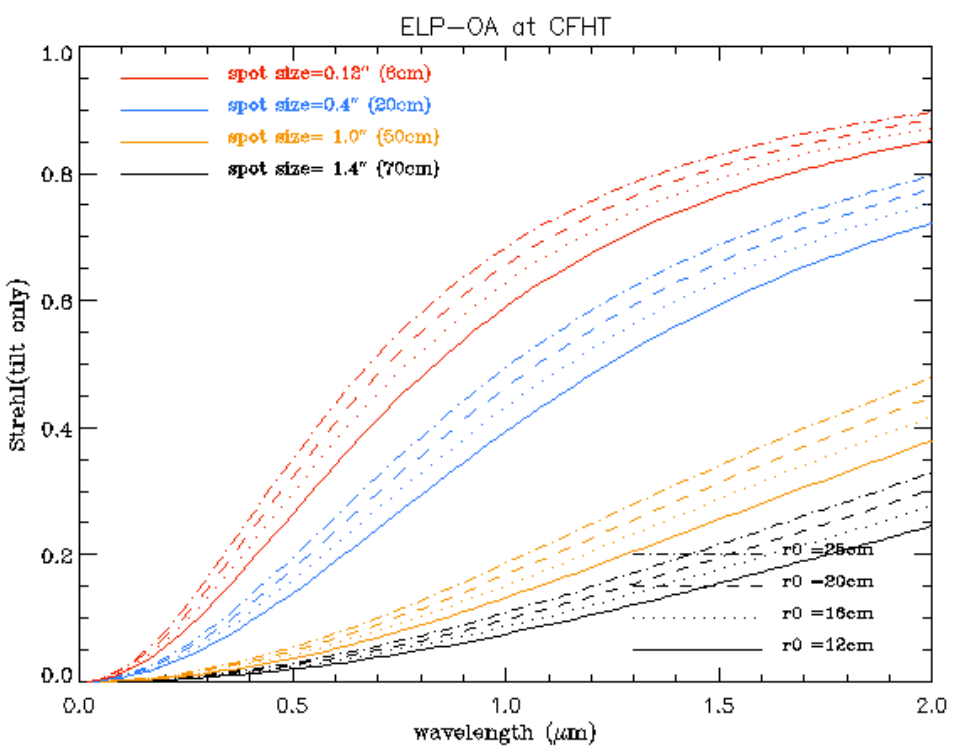

Figure 3: Strehl attenuation as a function of wavelength for various spot sizes (colored line bundles) and r0 (dotdashing). A spot size $<0.4$ " is required for meaningful correction at visible wavelengths.

\subsubsection{Fresnel propagation of laser beam through thick sodium layer}

Most LGS systems use collimated beams and do not try to focus on the sodium layer. However, in the case of the polychromatic laser guide star, the spot size is paramount. Figure 4 shows the spot size at the mesosphere for a diffraction limited launch telescope and for a naturally divergent (collimated Gaussian) beam. This shows the necessity of obtaining a diffraction limited spot at the mesosphere, if the spot is to be smaller than $0.5 "(1 \mathrm{~m}=2.1 "$ at $100 \mathrm{~km})$. However, even a focused beam will not provide the diffraction limited $\lambda / \mathrm{D}$ FWHM, as the sodium layer has a (timevarying) thickness averaging $10 \mathrm{~km}$. Analytical calculation of the spot size, depth of focus and diffraction ring intensity (Urey, 2004 [13]) were carried out, assuming a perfect wavefront (no turbulence):

$$
I(T, r, z)=\frac{8 \pi a^{2}}{\lambda^{2} z^{2} T^{2} P_{\text {beam }}}\left|\int_{0}^{1} e^{-\frac{\rho^{2}}{T^{2}}} \exp \left[\frac{i \pi \rho^{2} a^{2}}{\lambda}\left(\frac{1}{z}-\frac{1}{R}\right)\right] J_{0}\left(\frac{\pi \rho r}{z}\right) \rho \cdot d \rho\right|^{2}
$$

where $T$ is the Gaussian truncation factor $(T<<1$ : Gaussian beam, $T>>1$ : top hat), $r$ is the radial coordinate at the focus, $z$ is the axial coordinate, $a$ is the aperture radius, $R$ is the distance along the optical axis of the beam waist, $\rho=r / a$ is the normalized aperture coordinate, $\lambda$ is the wavelength and $P_{\text {beam }}=1-\exp \left(-2 / T^{2}\right)$ is the power transmitted by the truncated Gaussian. This has been solved numerically for a range of values of $T$ and $D(=2 a)$ and is shown in Figure 4.

To carry out the analysis further, we pick $D=0.5 \mathrm{~m}$ and $T=0.9$ (so that $P_{\text {beam }}$ is close to 1), and we use a time sample of the vertical sodium distribution obtained by LIDAR at AEOS on Haleakala in Maui on August $11^{\text {th }}$, 2004 (http://conrad.csl.uiuc.edu/Research/Maui/, shown on Figure 5). By multiplying the diffraction pattern by the sodium density, we can estimate the time evolution of the spot size and vertical profile. We find that the spot shape does not change very much as seen from the ground but it does roughly double in size (from 0.13 " to 0.3 ") with respect to the diffraction limit at the best focal plane. This is shown in Figure 4. Another effect that was brought to attention by this work is that the distance of the laser guide star can vary by a few $\mathrm{km}$ if one looks at the center of gravity of the 
elongated spot, or by $>10 \mathrm{~km}$ if one looks at the brightest spot; the error in focus can therefore be considerable. A full treatment including turbulence has not been carried out as it would require the full Fresnel propagation to be simulated for each phase screen realization.
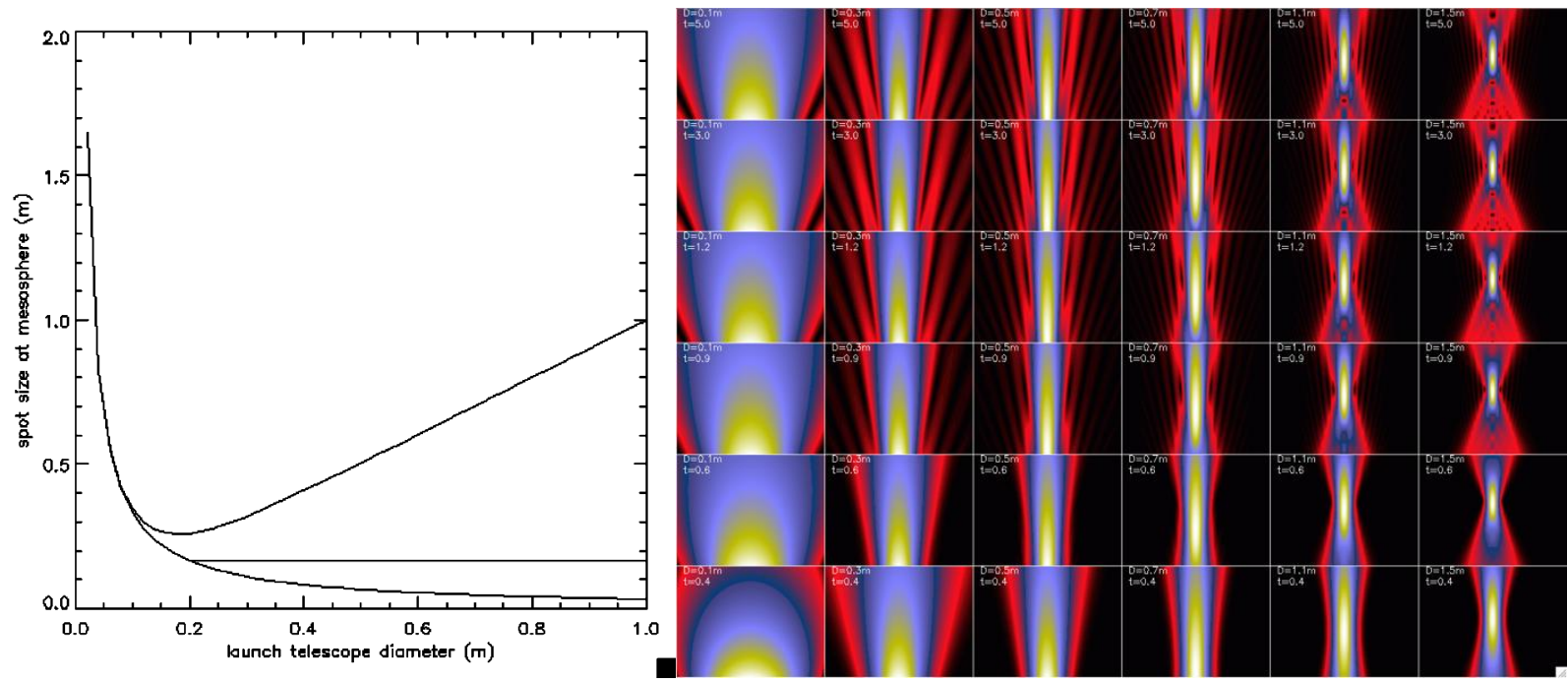

Figure 4 (left): spot size as a function of launch telescope diameter; bottom curve is diffraction limited, middle (flat) curve is seeing limited and top curve is natural divergence of Gaussian beam. Right: Fresnel diffraction through focus for various launch telescope diameters and Gaussian clipping. The scale is 1 meter (2") along the $x-$ axis and $40 \mathrm{~km}$ centered around $90 \mathrm{~km}$ on the $y$-axis of each panel. Decreasing telescope size left to right, Gaussian truncation decreasing top to bottom.

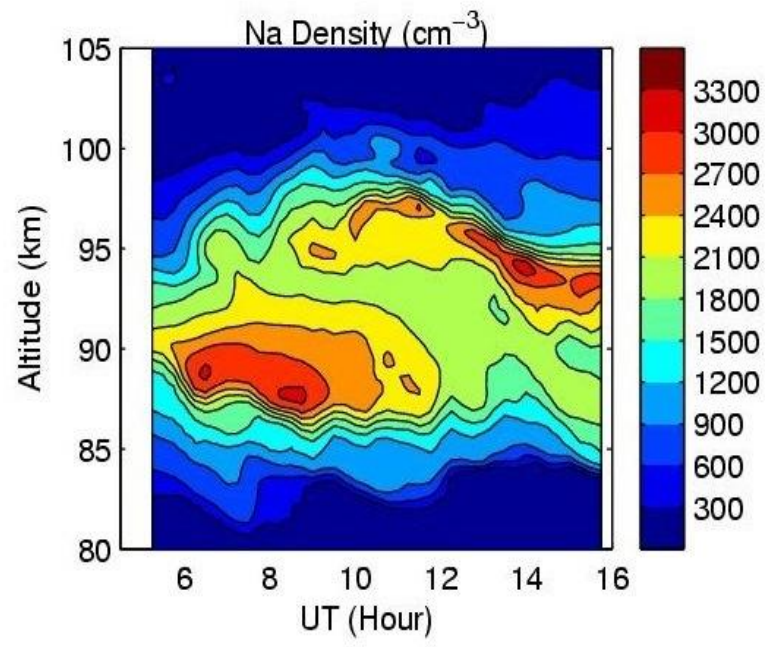

Na Lidar, Maul

August 11, 2004

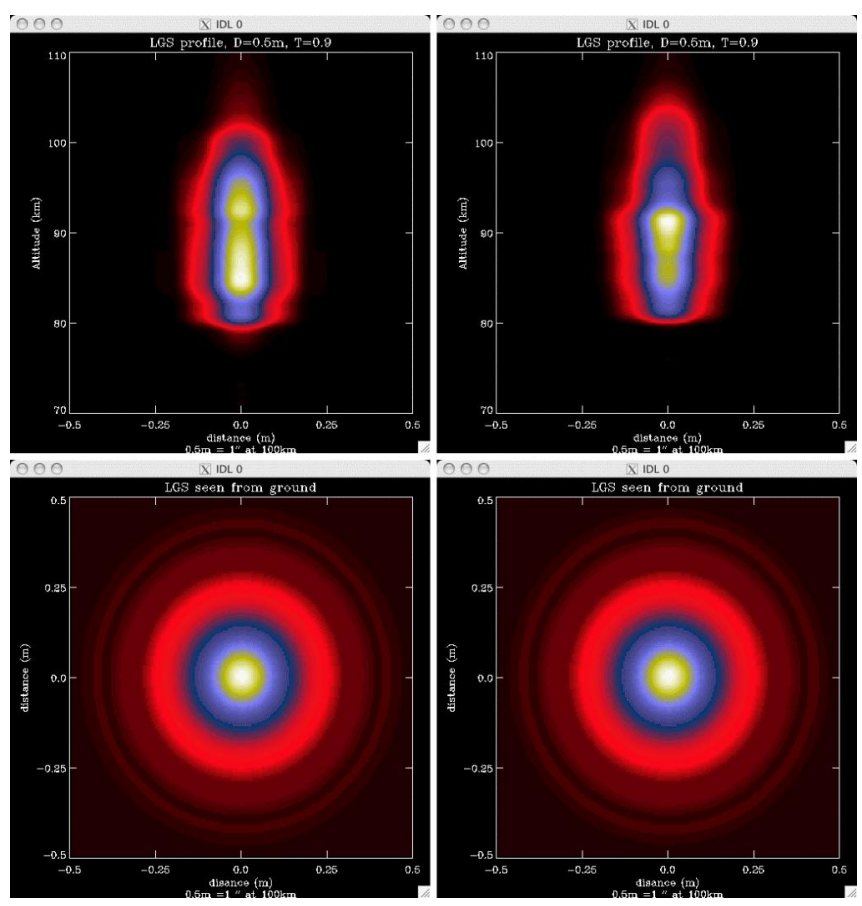

Figure 5: Left, vertical sodium distribution obtained by LIDAR at AEOS on Haleakala, Maui. Right, examples of LGS variation due to sodium density. Top: vertical cuts of the LGS, taking into account Fresnel diffraction and the sodium density (left at 10UT, right at 14UT), linear scale. Bottom: Same but seen from the ground on-axis (vertical average). Note that although the vertical distribution changes, the FWHM is quite insensitive but is broadened by a factor 2 with respect to $\lambda / \mathrm{D}$. The scale is square root. 


\subsubsection{Open loop measurement}

Because the polychromatic tip-tilt sensor is sensitive to a chromatic effect but the corrective tip-tilt mirror is achromatic, the correction is not propagated to the sensor and thus, the sensor works in open loop. While this is does not preclude efficient correction (see e.g. Andersen et al., these proceedings [1]), it requires a very high level of calibration both of the sensor and the mirror as there is no feedback of any errors and the dynamic range of the sensor has to be large (as it works off its null position). Also because the this sensor is only sensitive to chromatic effects, vibrations, drifts or jumps of the telescope will not be measured and the telescope guiding must come from other sources: a seismometer is required to control the telescope's attitude in an inertial reference frame and a slow guider (using time averaged multiple sources in the field, for example) eventually ties back to the sky.

\subsubsection{Two-color experiment}

In order to evaluate the accuracy achievable with a given number of photons and estimate the temporal error in a real setting, an experiment was carried out at the CFH telescope using the adaptive optics bonnette. The beam is split by a bamsplitter cube and is brought to focus on two Prosilica GE680 cameras each driven by its own PC. The two cameras are synchronized with the wavefront sensor pulses so that images are acquired simultaneously and can also be matched up to wavefront sensor measurement. Each camera has two filter wheels with ND filters (to match the flux) and narrow band filters (one in the UV band, one in the near IR). The experimental setup is show on Figure 6.

Due to the large amount of data generated by these two cameras, two acquisition modes were intended: with a $480 \times 480$ raster, the highest frame rate achievable was $25 \mathrm{~Hz}$. In this mode, the idea was to obtain data with the $\mathrm{AO}$ system in open loop, so that the absolute and differential tip-tilt could be measured directly from both cameras. The pixel scale was 10 mas, but even with an almost 5" field of view, the star image wandered off the cameras thus affecting the centroid estimation (Figure 6, top right). In the second mode, the raster was $200 \times 200$, and frame rates of up to $200 \mathrm{~Hz}$ were achieved. Here the idea was to stabilize the images using adaptive optics correction and correlating the tip-tilt recorded in the circular buffers with the differential tip-tilt measured from the cameras. Again the small field of the cameras meant that part of the flux wandered off the cameras, even in closed loop, affecting the estimation of the centroid (Figure 6, bottom right).
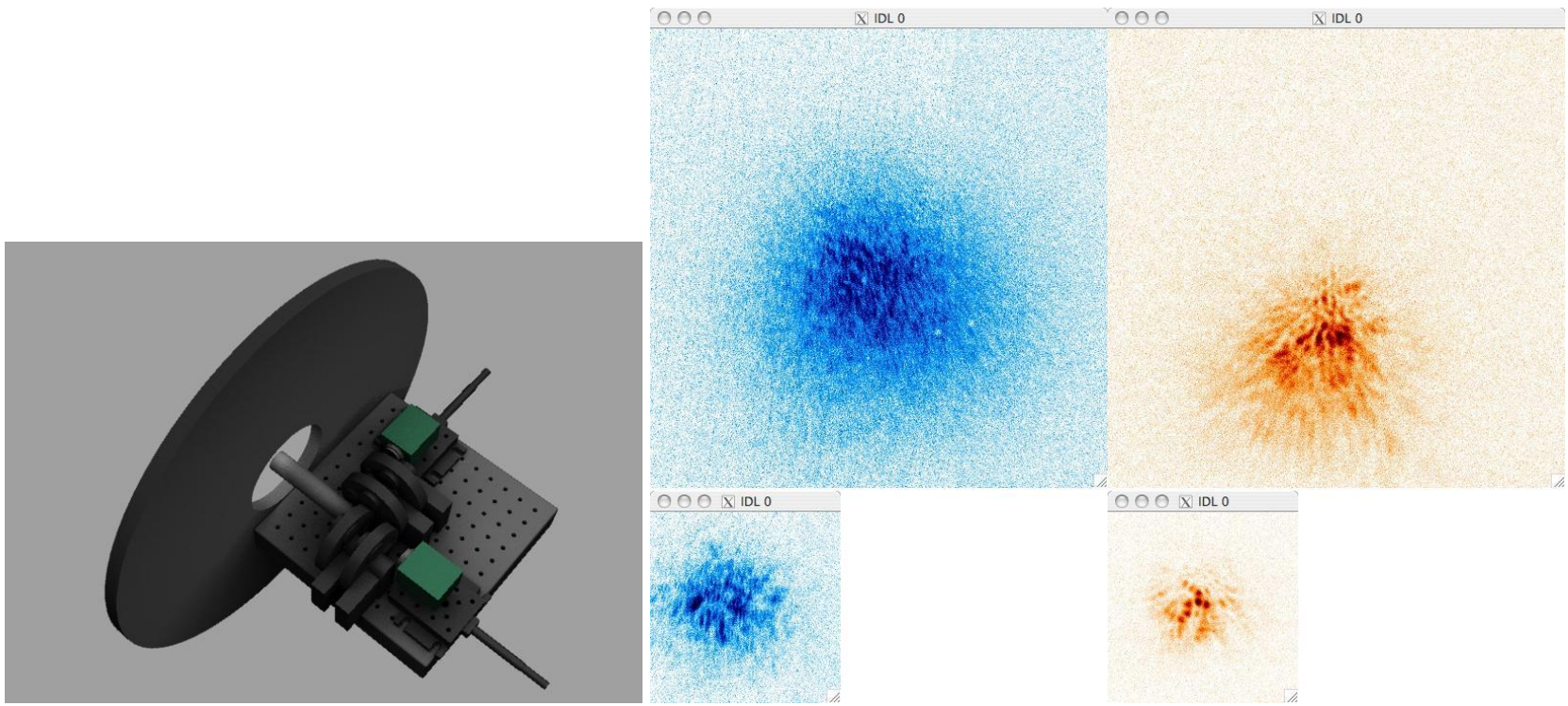

Figure 6: left, layout of twocolor experiment at the focus of AOB showing the two cameras. Right: results on Sirius in open loop (top, field:4.8”)) and closed loop (bottom, field:2") with the same plate scale (10mas/pix). As can be seen on all the images, clipping at the edges will affect the centroid estimation.

The plate-scale was chosen to provide Nyquist sampling on the UV camera and to try to improve the estimation of the centroid (see section 3.2.5.1) by wavefront reconstruction. However, the field was too small to produce meaningful results. Therefore new observations with a larger plate scale (25mas/pix) are planned for the summer of 2008. 


\subsubsection{Centroid improvement schemes}

As we have seen so far, the requirements on the accuracy of the differential tip-tilt are such that a seeing limited spot would require unreasonable amounts of laser power.

\subsubsection{Post-processing schemes}

The centroid is the optimal estimator of tip-tilt in the case of a Gaussian spot with infinite sampling; however, there may be some ways to improve on this if we use some a priori knowledge or use information contained in the speckle pattern or beam shape. Since the two monochromatic images have gone through the same phase screen but are realized at different wavelengths, phase diversity-like techniques could potentially be applied to estimate the wavefront more accurately than simple centroiding, although at the expense of real time computing power. Work by Rondeau et al., (2006 [10]) show promise in this direction.

\subsubsection{Mesospheric interference fringes}

The idea of producing interference fringes in the mesosphere has also been suggested, as the fringe pattern would move around due to the turbulence, but as long as each launch telescope is smaller than $r_{0}$, the overall fringe pattern's contrast would not be affected. The drawbacks of this method are that:

1. The fringes are blurred on the way back down (although the high order AO system on the primary would correct for some of this at $589 \mathrm{~nm}$, correction would be minimal at $330 \mathrm{~nm}$ ) and

2. Seen from the edge of the pupil, the finite thickness of the sodium layer means that the top of the center fringe would overlap with the bottom of the bright next fringe, thus reducing the fringe contrast. Quantitatively, a $10 \mathrm{~km}$ vertical fringe at $100 \mathrm{~km}$ subtends a 0.37 " angle when seen from 1.8 meter off-axis $(\mathrm{D} / 2)$. Full Fresnel propagation shows this effect in Figure 7. This means that for the fringe spacing has to $>0.4$ " if the contrast is not to be affected by the finite thickness of the sodium layer.
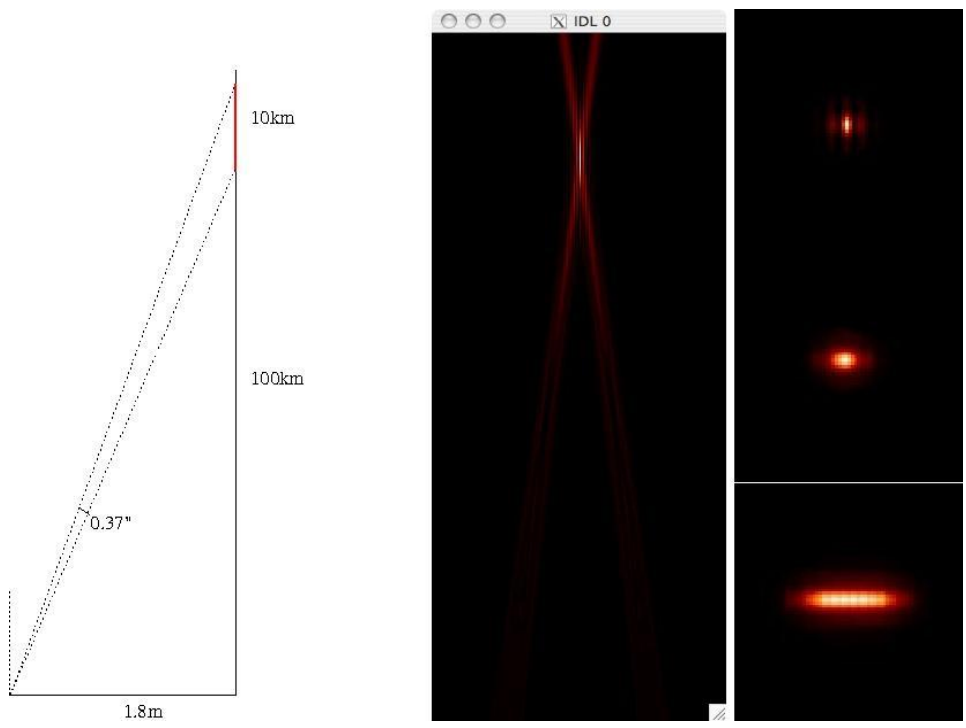

Figure 7: Fresnel propagation simulation of fringes in the mesosphere. The three panels on the right the fringe blurring at different off-axis positions (top: $0 \mathrm{~m}$, middle:0.6m, bottom: $1.8 \mathrm{~m}=\mathrm{D} / 2$ )

The fringe spacing can of course be adapted to be larger than the projected fringe size, multiple launch telescopes can be used to control the secondary lobes and temporal gating could also be used to disentangle the fringe pattern, so this solution may still show some promise, although not in its most basic implementation described here.

\subsection{Slow guiding}

The slow guiding should pose no particular problem, as the integration times can be on the order of a minute and the tiptilt can be well averaged. This can be further improved if a few sources in the field are averaged, further reducing any non-stationarity that may be present in the atmosphere (e.g. dominant wind direction). By using a CMOS as the 
scientific detector, non destructive reads of the array during the integration on the science object can also be used to fine-tune the slow guiding.

\subsubsection{Pendular seismometer}

A seismometer based on the design of Tokovinin (2000 [12]) was designed and built at CFHT. By mounting one such device along each axis (hour angle and declination) of the telescope, the telescope's attitude can be measured and corrected for with respect to an inertial frame of reference. This device consists of two $2.2 \mathrm{~kg}$ masses mounted 1 meter apart on a carbon fiber rod. Two extremely sensitive LVDTs manufactured by Schaevitz, mounted on opposite ends, sense angular deflections. The pendulum position is maintained at null by a low bandwidth servo system. A custom voice coil motor serves as the drive mechanism. The device was tested and provides milli-arcsecond sensitivity (Figure 8 ); this is within the level of accuracy required for open loop guiding.

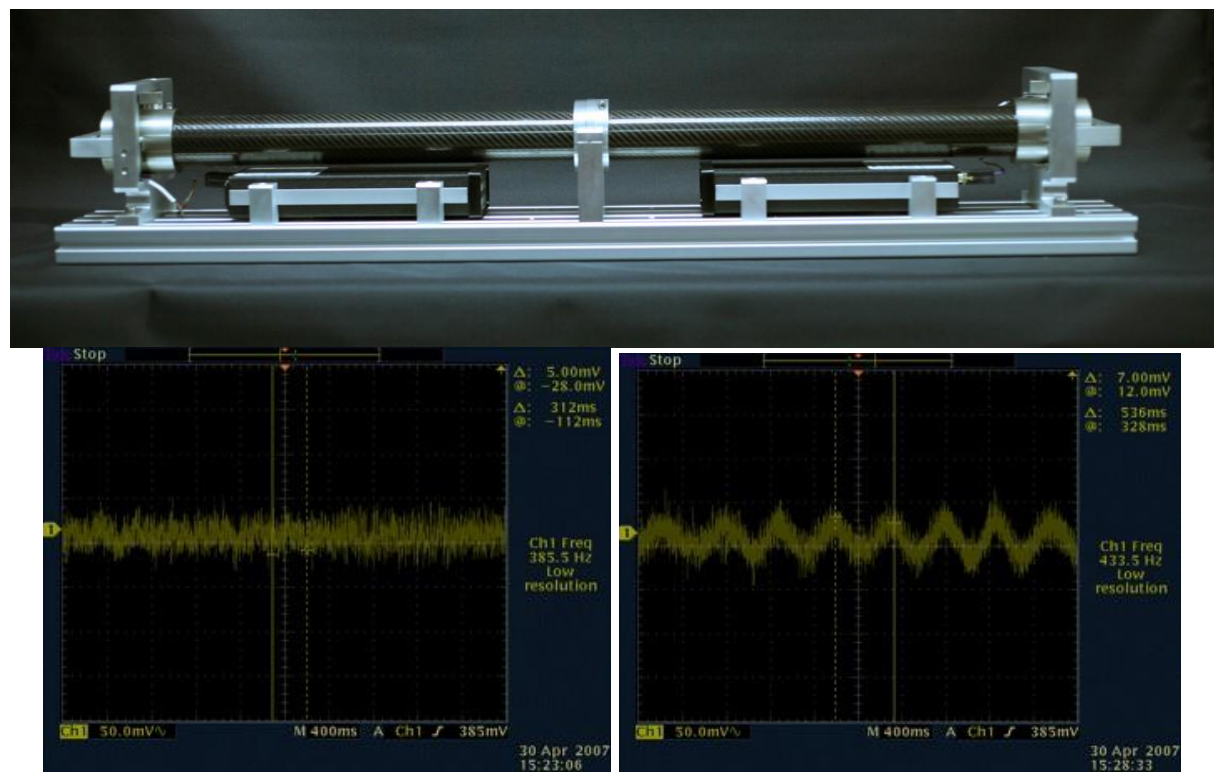

Figure 8, top: Photograph of pendular seismometer. Bottom left: With the seismometer mounted on the Caisson Central of the telescope, the hydraulic oil system powered and the telescope servoing, the peak-to-peak detected motion is on the order of 70mas. Bottom right: With the telescope moving at 0.1 arcsec/second in the declination axis, the peak-to-peak motion increased to $100 \mathrm{mas}$ and a $2.5 \mathrm{~Hz}$ vibration is visible.

\section{Feasibility study}

VASAO was suggested first and foremost for its astrophysical potential, and because it provides a scientific niche. Furthermore, a telescope such as CFHT is in an ideal position to study innovative concepts at the forefront of technology. As is often the case, greater rewards also come at either larger cost or larger risk. The polychromatic tip-tilt determination is an elegant solution but it is neither simple nor risk-free. Therefore a large effort has gone into the feasibility study of this concept. This has taken the guise of simulations and computations (as described in section 3), but also of a detailed error budget and some experiments.

\subsection{Error budget}

A detailed error budget was developed and is shown on Figure 9. It is immediately apparent that the major item on the error budget is the polychromatic tip-tilt error. The goal of the error budget was $122 \mathrm{~nm}$ rms which provides a Strehl ratio of 0.3 at $700 \mathrm{~nm}$. Using various spot sizes and laser power (and some generous assumptions), the residual tip-tilt is $315 \mathrm{~nm}$ rms for a 0.7 " spot size and $100 \mathrm{~W}$ laser and even in the case of a 0.3 " spot size (and 100 W laser), it remains well above the goal, at $184 \mathrm{~nm}$ rms. We note that it is the simultaneous requirement of all-sky AND visible that prevents us from reaching our goal. If we were to re-scope the project to near-infrared all-sky, then we can see from the error budget that a Strehl of 0.3 at 1.6 microns, which would require on the order of $300 \mathrm{~nm} \mathrm{rms,} \mathrm{is} \mathrm{achievable.}$ 


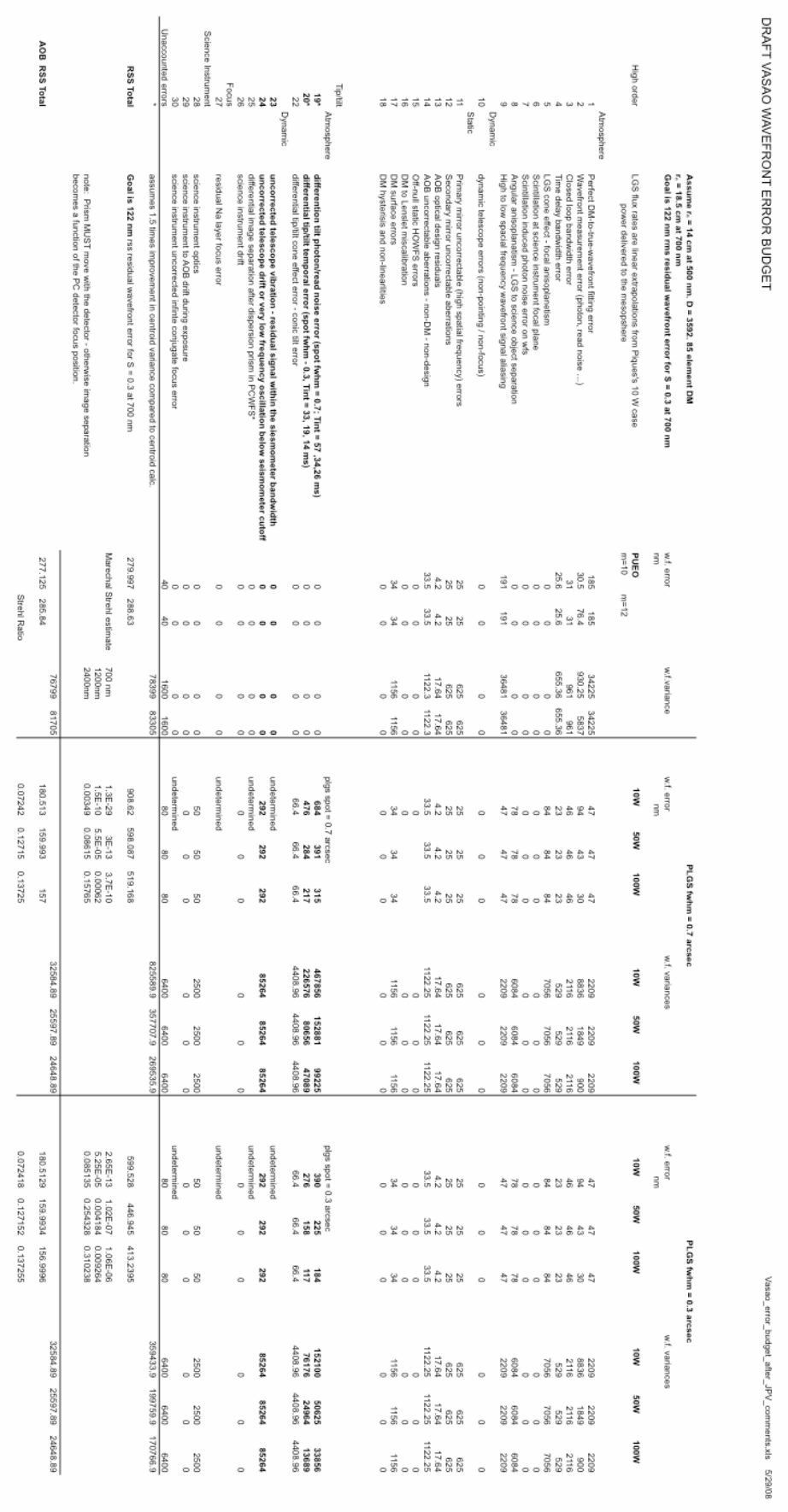

Figure 9: VASAO error budget. Various cases of spot size and laser power are considered. The differential tiptilt measurement is the dominant term.

\subsection{Experiments}

We have already described the FlyEyes experiment (section 3.1.1) and the two-color experiment (section 3.2.4). The seismometer (section 3.3.1) needs to be further tested on the telescope and may yet find uses to solve the telescope's declination axis oscillation issues. 
One major contentious issue is the actual return flux per watt of laser power propagated to and back from the sodium layer. In the polychromatic LGS scheme more than one line needs to be excited, or ideally a cascade through the energy levels would provide the multiple wavelengths required for the polychromatic differential measurement. Various excitation schemes have been proposed (Foy et al.. 1995 [4], Pique et al., 2006 [8]) but exciting the UV-D2 line at $330 \mathrm{~nm}$ and measuring the return flux at the various wavelengths is an experiment that would provide a definitive answer as to whether this method is viable. A campaign is scheduled for the end of 2009, where a dye laser tuned to $330 \mathrm{~nm}$ will be used in parallel with the Gemini, Keck and hopefully the Subaru lasers as well as a LIDAR, to provide a direct comparison and quantitative estimate of the effective cross section of sodium and return flux per watt.

\section{Conclusion}

We have presented the VASAO (Visible All-Sky Adaptive Optics) concept. The high order adaptive optics system, PUEO HOU, poses no particular difficulty: a curvature system with 85 sub-apertures provides adequate performance in the visible, with negligible focal anisoplanatism. The main difficulty comes from the determination of the tip-tilt and specifically, if the LGS is seeing limited, the laser power required to measure the centroids with sub-milliarcsecond accuracy is prohibitive. Various schemes to improve the accuracy on the centroids (from up-launch AO, to mesospheric interference fringes) have been studied but no simple concept provides an obvious solution.

Experiments are being carried out to quantitatively estimate the accuracy on the centroids with a given number of photons and the real number of return photons per watt of propagated laser power.

A re-scope of the project to provide near infrared diffraction limited all sky coverage is seen as the most realistic and cost-effective way forward. Indeed many of the astrophysical applications (binarity in the solar system, YSOs, followup of deep fields individual objects, etc.) benefit more from the unique aspect of all-sky coverage rather than the enhanced resolution (comparable to that of 8-10meter telescopes in the near-IR) or visible domain per se. Visible LGS adaptive optics is also a niche, both scientific and technological that would provide unique capabilities to our communities. There would be no major difficulty in implementing such a system, and would be a natural step-toupgrade on the way to VASAO.

\section{References}

[1] Andersen D.R., Fischer M.D., Conan R., Fletcher M., Véran J.-P., 2008, These Proceedings, 7015-16, “VOLT: the Victoria Open Loop Testbed"

[2] Beletic, J.W., Dorn Re.J., Craven-Bartle T., Burke B., 4th ESO CCD Workshop Proceedins, 283. "A new CCD designed for curvature wavefront sensing"

[3] Dougados C., Cabrit S., Lavalley C., Ménard F., 2000, A\&A 357, 61. "T Tauri stars microjets resolved by adaptive optics"

[4] Foy R., Migus A., Biraben F., Grynberg G., McCullough P.R., Tallon M., 1995, A\&AS 111, 569. "The polychromatic artificial sodium star: a new concept for correcting the atmospheric tilt."

[5] Ho K.Y., Cuillandre J-C., Lin C-J., Benedict T., Lai, O., Ward J., Salmon D., Luppino G., Beletic J., Dorn R., Puget P., Burke B., Wang S-Y., 2006, SPIE Proc. 6276, 43. "FlyEyes: integrating CCID-35 into PUEO AO system at CFHT"

[6] Lai O., 2002, ESO Conference and Workshop Proceedings 58, 343. "High order curvature Adaptive Optics revisited."

[7] Ménard F., Stapelfeldt K., Krist J., Duvert G., Padgett D., Burrows C., BAAS 31, 933. "The Circumbinary Disk of UY Aurigae: Combining Hubble Space Telescope and Adaptive Optics Images"

[8] Pique J.-P., ; Moldovan I.C., Fesquet V., Guillet de Chatellus H., Marc F., 2006, SPIE Proc. 6272, 106. "Polychromatic laser guide star using a single laser at $330 \mathrm{~nm}$ "

[9] Rigaut F., private communication, http://www.maumae.net/yao/aosimul.html

[10] Rondeau X., Thiébault E., Tallon M., 2006, SPIE Proc. 6272, 108. "Polychromatic phase retrieval with Kolmogorov self-adapting prior constraints".

[11] Schoöck M., Foy R., Tallon M., Noethe L., Pique J-P., 2002, MNRAS 337, 910. "Performance analysis of polychromatic laser guide stars used for wavefront tilt sensing".

[12] Tokovinin, 2000, MNRAS,316, 637. "Pendular seismometer for correcting telescope vibrations"

[13] Urey H., 2004, Applied optics 43, 620. "Spot size, depth of focus, and diffraction ring intensity formulas for truncated Gaussian beams".

[14] Veillet C., Lai O., Salmon D., Pique J-P., 2006, SPIE Proc. 6272, 79. "VASAO: visible all sky adaptive optics" 